\title{
openheart Drug-eluting stents in large coronary vessels improve both safety and efficacy compared with bare-metal stents in women: a pooled analysis of the BASKET-PROVE I and II trials
}

Louise Hougesen Bjerking, ${ }^{\oplus 1}$ Kim Wadt Hansen, ${ }^{1}$ Rikke Sørensen, ${ }^{2}$ Eva Prescott, ${ }^{1}$ Tor Biering-Sørensen, ${ }^{3}$ Raban Jeger, ${ }^{4}$ Christoph Kaiser, ${ }^{4}$ Matthias Pfisterer, ${ }^{4}$ Søren Galatius ${ }^{1}$

\begin{abstract}
- Additional material is published online only. To view please visit the journal online (http://dx.doi.org/10. 1136openhrt-2018-000986).
\end{abstract}

To cite: Bjerking $\mathrm{LH}$, Hansen KW, Sørensen R, et al. Drug-eluting stents in large coronary vessels improve both safety and efficacy compared with bare-metal stents in women: a pooled analysis of the BASKET-PROVE I and II trials. Open Heart 2019;6:e000986. doi:10.1136/

openhrt-2018-000986

Received 11 December 2018 Revised 20 February 2019 Accepted 26 April 2019

Check for updates

(C) Author(s) (or their employer(s)) 2019. Re-use permitted under CC BY-NC. No commercial re-use. See rights and permissions. Published by BMJ.

For numbered affiliations see end of article.

Correspondence to Dr Louise Hougesen Bjerking; louise_bjerking@hotmail.com

\section{ABSTRACT}

Background Drug-eluting stents (DES) have proven superior to bare-metal stents (BMS) in terms of safety and efficacy. However, inference to the female subgroup has been limited by low enrolment rates of women in clinical trials. The objective of this study was to investigate the safety and efficacy of DES versus BMS in women and men. Methods In a pooled analysis of two all-comers randomised trials (Basel Stent Kosten-Effektivitäts TrialProspective Validation Examination (BASKET-PROVE) and BASKET-PROVE II) ( $n=4605)$, we examined safety and efficacy of DES versus BMS according to sex. Patients were followed 2 years for a composite endpoint of cardiac death, non-fatal myocardial infarction (MI) and clinically driven target-vessel revascularisation not related to MI. Results Among the 1076 women and 3529 men included in the analysis, $65.6 \%$ of the women and $67.2 \%$ of the men were randomised to receive DES. At baseline, men had more complex coronary artery disease than women. After 2 years, DES reduced rates of major adverse cardiac events (MACE) compared with BMS in both women (cumulative incidence, $6.1 \%$ vs $14.7 \%$; adjusted HR 0.36 ( $95 \% \mathrm{Cl} 0.24$ to 0.54$)$ ) and men (7.7 vs $12.1 \%$, HR 0.62 $(0.50$ to 0.77$)$ ), although the reduction in MACE rates was more pronounced in women (adjusted $p=0.02$ for sexstent interaction). Event rates were lower in DES for both safety and efficacy outcomes, with the largest effect seen for non-MI TVR, in both women (2.3 vs $9.2 \%$, adjusted HR 0.24 (0.13 to 0.44)) and men (4.0 vs $7.8 \%$, adjusted HR 0.48 (0.36 to 0.64)) (adjusted $p=0.049$ for sex-stent interaction).

Conclusions In patients requiring stenting of large coronary arteries, DES were associated with improved safety in women and superior efficacy in both sexes as compared with BMS.

\section{INTRODUCTION}

Drug-eluting stents (DES) are associated with lower rates of target vessel revascularisation $(\text { TVR })^{1-4}$ compared with bare-metal stents (BMS), possibly with a particular benefit in

\section{Key questions}

What is already known about this subject?

- Drug-eluting stents (DES) are associated with lower rates of target vessel revascularisation compared with bare-metal stents (BMS) in both genders - possibly with a particular benefit in women. However, inferences on efficacy and safety of DES versus BMS have been difficult to study in female subgroups as clinical trials on coronary artery disease often enrol a low proportion of women.

What does this study add?

- This study conducted on patients requiring stenting of large coronary arteries reports an adjusted comparison of BMS versus DES in women with a male comparator group. DES were associated with improved safety in women and superior efficacy in both genders compared with BMS. These findings consolidate prior findings that DES are safe and effective in women but indicate a safety benefit not observed in men.

How might this impact on clinical practice?

- Our findings suggest that DES may be regarded as the stent of choice for women.

women. ${ }^{5-7}$ However, it has been difficult to compare both the efficacy and safety of DES versus BMS in female subgroups as clinical trials often enrol a low proportion of women. Recently, a large pooled study of female participants showed DES to be safe and more effective, ${ }^{8}$ but by design a male comparator group was not included. The Basel Stent Kosten-Effektivitäts Trial-Prospective Validation Examination (BASKET-PROVE (BP) $)^{9}$ and the BASKET-PROVE II (BPII) ${ }^{3}$ trials were large all-comers randomised stent trials comparing DES versus BMS in patients with large coronary vessels. In the present study 
we investigated the safety and efficacy of DES versus BMS in both women and men in a pooled analysis of BP and BPII.

\section{METHODS}

\section{Study population}

A more comprehensive description of the $\mathrm{BP}$ and the BPII trials can be found elsewhere. ${ }^{39-11}$ In brief, the BP (clinicaltrial.gov ID: NCT01166685) and BPII (ISRCTN registry number: ISRCTN72444640) trials were large prospective all-comers randomised trials comparing DES versus BMS. Patients with chronic or acute coronary disease undergoing angiography and stenting of large coronary arteries at least $3.0 \mathrm{~mm}$ in diameter were included. Cardiogenic shock, in-stent restenosis or thrombosis, unprotected left main coronary artery or bypass-graft disease, planned surgery within 12 months, need for oral anticoagulation or increased bleeding risk, known intolerance to or suspected noncompliance with long-term antiplatelet drug therapy, history of transient ischaemic attack or stroke, and impossible follow-up were exclusion criteria. In BP the patients were equally randomised 1:1:1 to receive a BMS, a first generation DES or a second generation DES. They received aspirin indefinitely and clopidogrel for 1 year as antithrombotics. In BPII the patients were randomised 1:1:1 to receive BMS or one of two second-generation DES (a second-generation durable-polymer DES or a biodegradable-polymer DES). Aspirin and prasugrel were prescribed as antithrombotic medication. Prasugrel was prescribed in a risk-adjusted dose for 12 months, except for patient with stable angina pectoris (SAP) treated with BMS, who received prasugrel for 1 month. In both BP and BPII, the patients were followed up for at least 2 years to investigate late events.

\section{Endpoints}

The primary endpoint in the present study was a combined safety and efficacy endpoint of major adverse cardiac events (MACE) including cardiac death, non-fatal myocardial infarction (MI) and non-MI TVR at 2 years. Our secondary outcomes were (1) a combined safety endpoint consisting of cardiac death and non-fatal MI, and (2) an efficacy endpoint defined as non-MI TVR. Cardiac death was defined as any death without a clear non-cardiac cause, and non-fatal MI as a clinical event with characteristic ECG or enzymatic changes, not leading to death. Non-MI TVR was any revascularisation of the primary target vessel not associated with MI. ${ }^{39-11}$ An independent committee adjudicated all critical events in a blinded fashion, with exception of the last third of endpoints in $\mathrm{BP}$ that were adjudicated without blinding.

\section{Statistics}

Discrete data are presented as counts and percentages, and continuous data as median and IQR. $\chi^{2}$ test was used to compare categorical data and non-parametric MannWhitney U-test to compare continuous data. We analysed the cumulative incidence of endpoints at 2 years by using
Kaplan-Meier estimator. The log-rank test was used to evaluate sex specific differences. The safety (cardiac death or non-fatal MI) and efficacy (non-MI TVR) of DES versus BMS in men and women with large coronary vessels were examined with adjusted Cox proportional hazard regression models. The Cox model for MACE was adjusted for age, clinical presentation (ST-elevation myocardial infarction (STEMI), non-ST-elevation acute coronary syndrome (NSTE-ACS) or SAP) and a history of current smoking, diabetes mellitus, hypertension or hypercholesterolaemia. The Cox models for the secondary endpoints were only adjusted for age and type of stent according to few endpoints. Interaction between sex and type of stent (DES vs BMS) was addressed using a likelihood ratio test. The proportional hazards assumption was tested quantitatively by testing Schoenfeld residuals for time dependency and visually by $\log$-log curves. The analyses were done by the intention-to-treat principle. Sex-specific analyses for each individual BP and BPII study has been specified a priori, ${ }^{911}$ while the present pooled study was designed and performed post hoc. All statistical tests were made with Stata/IC V.14.0 (StataCorp) and had a two-sided significance level of 0.05 .

\section{RESULTS}

In total, 4605 patients (23.4\% women) were included in the analysis (figure 1). DES and BMS were equally distributed between the sexes with $65.6 \%$ of the women and $67.2 \%$ of the men randomised to receive a DES. Mean follow-up time was 732 (IQR (701-757)) days.

\section{Baseline characteristics}

Table 1 shows the baseline characteristics.

Women were older and presented with more hypertension, heart failure and renal disease than men, while risk factors such as current/former smoking, prior MI and prior revascularisation were more frequent in men. There were no significant differences in patient characteristics between the two stent type groups (BMS and DES) in neither men nor women. No significant differences between sexes in clinical presentation with respect to distribution of STEMI, unstable (NSTE-ACS) and SAP were seen. In terms of clinical presentation, NSTE-ACS was more prevalent in the DES group among women compared with the BMS group. STEMI was a more frequent presentation among men in the DES group versus the BMS group. Men tended to have more severe coronary disease in terms of more multivessel disease and chronic total occlusion than women (online supplementary table 1).

Table 2 lists procedure-related characteristics.

Compared with women treated with DES, significant more women treated with BMS had bifurcational lesions $(\mathrm{p}=0.037)$. The length of stent per lesion was significant larger in men in the DES group compared with men in the BMS group $(p=0.009)$. No other differences in the 


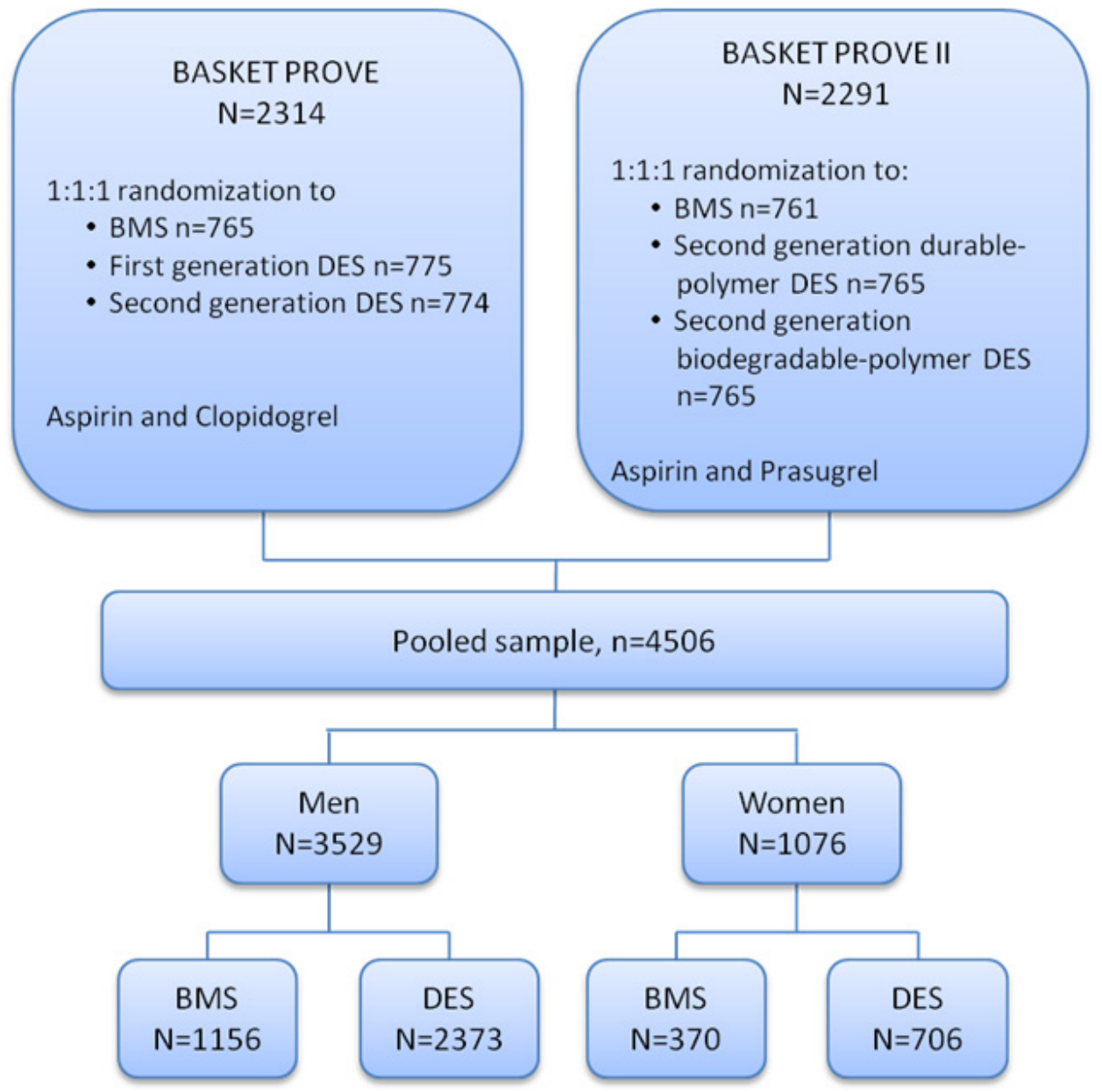

Figure 1 Flow chart showing the pooled sample of participants from the BASKET-PROVE and BASKET-PROVE II trials. BASKET-PROVE, Basel Stent Kosten-Effektivitäts Trial-Prospective Validation Examination; BMS, bare-metal stent; DES, drugeluting stent.

procedure-related characteristics were seen between the two stent groups in either sex.

\section{Efficacy and safety}

DES significantly reduced the risk of MACE at 2 years compared with BMS in both women and men, and the cumulative incidence was significantly reduced in women (6.1 vs $14.7 \%$; adjusted HR, 0.36 (95\% CI 0.24 to 0.55$)$ ) and in men (7.7 vs $12.1 \%$; adjusted HR, 0.62 (95\% CI 0.50 to 0.77 )) (figures 2 and 3). There was a significant interaction between sex and type of stent (adjusted $\mathrm{p}=0.02$ ).

The stent-dependent difference in MACE was mainly driven by a difference in the efficacy endpoint non-MI TVR in both women and men, with a larger effect in women compared with men (2.3 vs $9.2 \%$; adjusted HR, $0.24(95 \%$ CI 0.13 to 0.44$)$ ) and (4.0 vs $7.8 \%$; adjusted HR, 0.48 (95\% CI 0.36 to 0.64$)$ ) (figure 3 ). The adjusted $\mathrm{p}$ value for interaction was 0.049 .

DES significantly reduced the relative hazard of the combined safety endpoint non-fatal MI and cardiac death compared with BMS with $52 \%$ in women (2.9 vs $5.3 \%$; adjusted HR, 0.48 (95\% CI 0.26 to 0.90$)$ ), and with a smaller and non-significant effect seen in men (3.3 vs 4.7\%; adjusted HR, 0.73 (95\% CI 0.51 to 1.04 )) (figure 3), but no significant sex-stent interaction was seen $(p=0.27$ for interaction). Similarly, the adjusted risk of cardiac death was significantly reduced in women (1.2 vs $2.8 \%$; adjusted HR, 0.36 (95\% CI 0.14 to 0.91 )), but not in men (1.4 vs 2.1\%; adjusted HR, 0.76 (95\% CI 0.44 to 1.27$)$ ) ( $\mathrm{p}$ value $=0.16$ for interaction). No significant differences in non-fatal MI were seen between DES and BMS, in men or women (figure 3). Event rates stratified by stent type and generation are summarized in Supplementary Table 2.

\section{DISCUSSION \\ Key findings}

This pooled analysis of patients requiring stenting of large coronary arteries demonstrated an improved safety of DES in women, but not in men. In line with prior findings, we found a lower risk for MACE in DES versus BMS in both sexes; mainly driven by lower rates of clinically driven TVR.

\section{Interpretations}

Although no sex-stent interaction was found for the combined safety endpoint of cardiac death and non-fatal 
Table 1 Baseline characteristics of the pooled study population

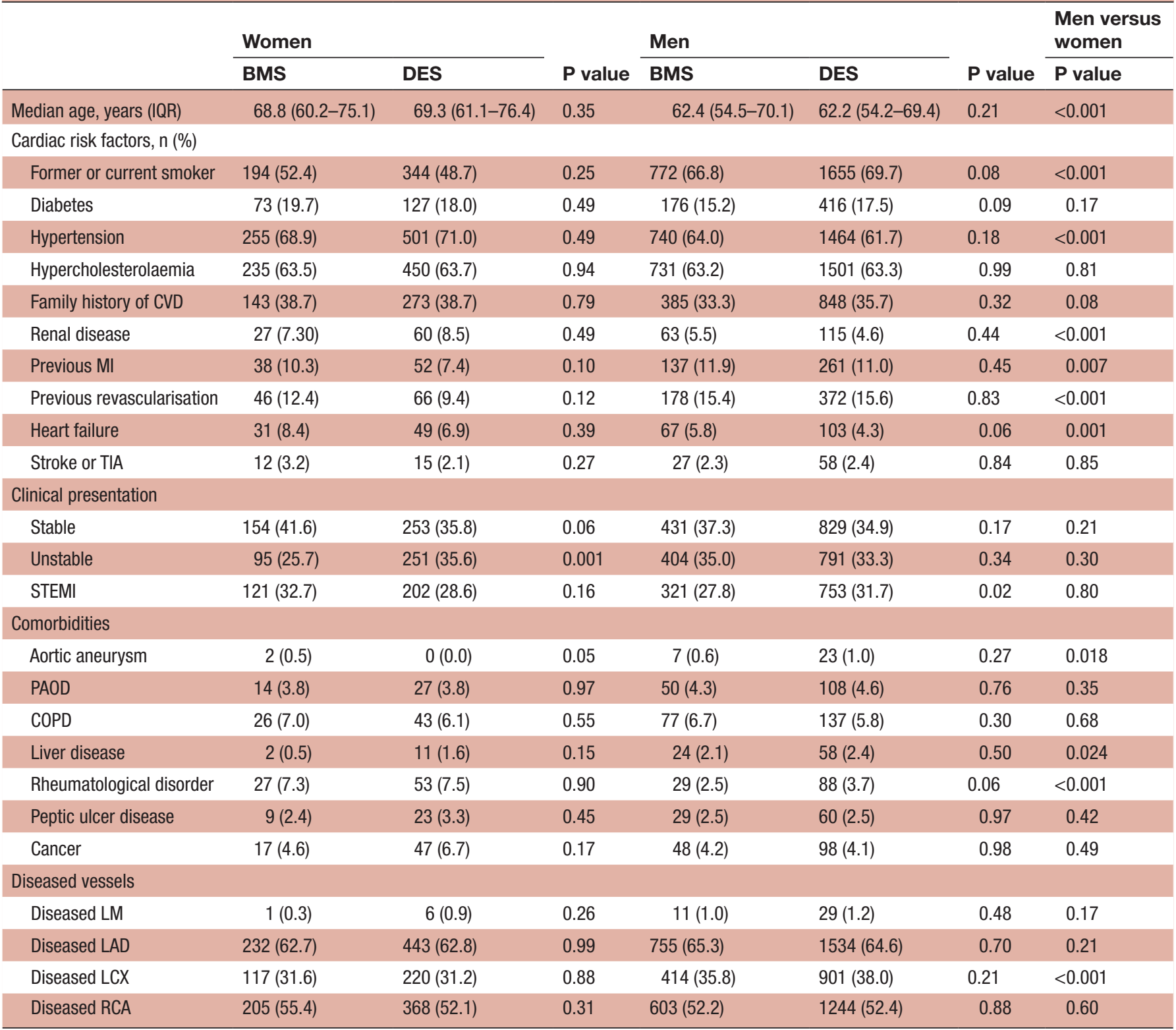

BMS, bare-metal stent; COPD, chronic obstructive pulmonary disease;CVD, Cardiovascular disease; DES, drug-eluting stent; IDDM, insulin-dependent diabetes mellitus; LAD, left anterior descending artery; LCX, left circumflex artery; LM, left main artery; MI, myocardial infarction; PAOD, peripheral arterial occlusive disease; RCA, right coronary artery;STEMI, ST-elevation myocardial infarction; TIA, transient ischaemic attack.

MI, we did observe a significant safety benefit in women treated with DES. Only a trend towards improved safety was found in men despite a larger sample size and more complex coronary artery disease (CAD). These findings suggest that factors other than vessel size and complexity of CAD were involved in the observed sex-related difference in safety and efficacy.

A substudy on the BP trial found a tendency towards a sex difference in MACE in the benefit of DES, but the study was underpowered to show a difference in safety. ${ }^{7}$ In a large pooled analysis $(\mathrm{n}=11557)$ of only female participants, Stefanini $e t a l^{8}$ found a differences in safety with respect to DES and BMS, consistent with our findings. The study did not include a male comparator group and a considerable heterogeneity among their study population was present. Our study contributes with data on a large homogeneous group of patients with $\mathrm{CAD}$. The NORSTENT study did not find a differences in death from any causes and nonfatal spontaneous MI after 6 years in a large population of patients treated with DES and BMS, respectively. ${ }^{4}$ However, gender subgroup analysis was not performed in this study.

On average women have smaller coronary vessels than men, and it has therefore been suggested that women might benefit more from DES. In our study, however, the diameter of the treated coronary vessels was above $3 \mathrm{~mm}$ in diameter in both women and men, and accordingly smaller vessels in women cannot explain the particular 
Coronary artery disease

Table 2 Procedure-related characteristics

\begin{tabular}{|c|c|c|c|c|c|c|c|}
\hline & \multicolumn{3}{|l|}{ Women } & \multicolumn{3}{|l|}{ Men } & \multirow{2}{*}{$\begin{array}{l}\text { Men vs } \\
\text { women } \\
\text { P value }\end{array}$} \\
\hline & BMS & DES & $P$ value & BMS & DES & $P$ value & \\
\hline No of patients & 370 & 706 & & 1156 & 2373 & & \\
\hline No of stented segments & 512 & 938 & & 1567 & 3164 & & \\
\hline \multicolumn{8}{|l|}{ Complexity of CAD } \\
\hline Multivessel disease & $146(39.5)$ & $249(35.3)$ & 0.20 & $480(41.5)$ & $992(41.8)$ & 0.90 & 0.0039 \\
\hline Bifurcational lesions & $31(8.4)$ & $35(5.0)$ & 0.037 & $82(7.1)$ & $162(6.8)$ & 0.82 & 0.041 \\
\hline Chronic total occlusions & $12(3.2)$ & $15(2.1)$ & 0.36 & $53(4.6)$ & $114(4.8)$ & 0.84 & 0.0020 \\
\hline Stents $<3.0 \mathrm{~mm}$ & $15(4.1)$ & $40(5.7)$ & 0.32 & $43(3.7)$ & $94(4.0)$ & 0.80 & 0.09 \\
\hline GPIlb/lla blocker use & $64(17.3)$ & 94 (13.3) & 0.096 & $197(17.0)$ & $454(19.1)$ & 0.14 & 0.005 \\
\hline \multicolumn{8}{|l|}{ Procedural characteristics } \\
\hline Treated segments per patient, no* & $1(1-2)$ & $1(1-1)$ & 0.091 & $1(1-2)$ & $1(1-2)$ & 0.52 & 0.73 \\
\hline Stents per patient, no* & $1(1-2)$ & $1(1-2)$ & 0.33 & $1(1-2)$ & $1(1-2)$ & 0.69 & 0.28 \\
\hline Total stent length, mm* & $22(15-34)$ & $18(15-33)$ & 0.50 & $22(15-35)$ & $23(15-36)$ & 0.16 & 0.04 \\
\hline Stent length per lesion, $\mathrm{mm}^{*}$ & $18(15-23)$ & $18(14-23)$ & 0.54 & $18(15-23)$ & $18(15-26)$ & 0.009 & 0.0035 \\
\hline Staged procedures & $21(5.7)$ & $41(5.8)$ & 1.00 & $57(4.9)$ & $136(5.7)$ & 0.37 & 0.77 \\
\hline Lesions with angiographic success & $495(96.7)$ & $923(98.4)$ & 0.051 & $1502(95.9)$ & $3052(96.5)$ & 0.34 & 0.006 \\
\hline
\end{tabular}

Numbers are counts (\%) unless otherwise stated.

*Median (IQR).

BMS, bare-metal stent; CAD, coronary artery disease; DES, drug-eluting stent.

female benefit of DES. The explanation may lie in differences in pathogenesis and pathology among men and women with $\mathrm{CAD}$. It has been shown that vascular inflammation is more pronounced in women with $\mathrm{CAD}$ than men, ${ }^{12}$ in particular a higher C-reactive protein raise has been observed in women with acute coronary syndrome. ${ }^{13}$ This is in accordance with the average female C-reactive protein measure which in general is higher than the male. $^{14}$

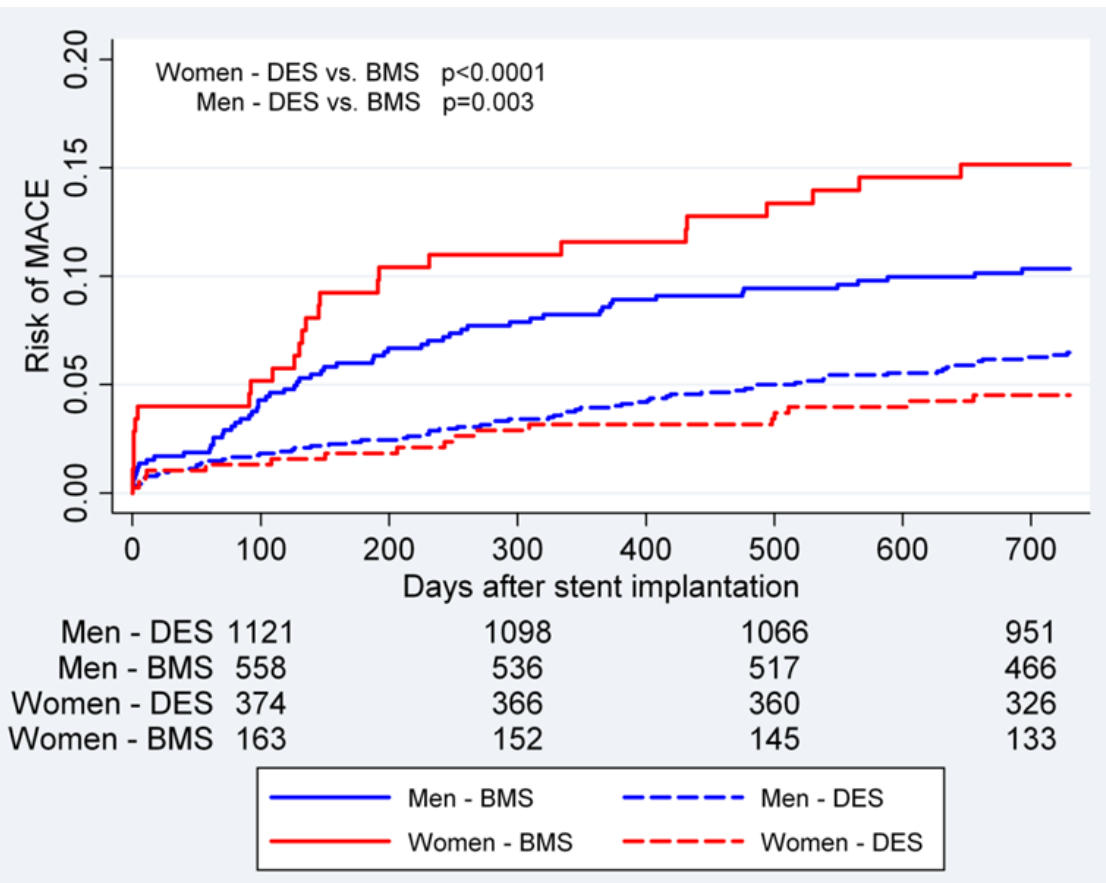

Figure 2 Kaplan-Meier survival curve showing the risk of MACE according to sex and type of stent. Full lines indicate BMS and dotted lines indicate DES. BMS, bare-metal stent; DES, drug-eluting stent; MACE, major adverse cardiac events. 


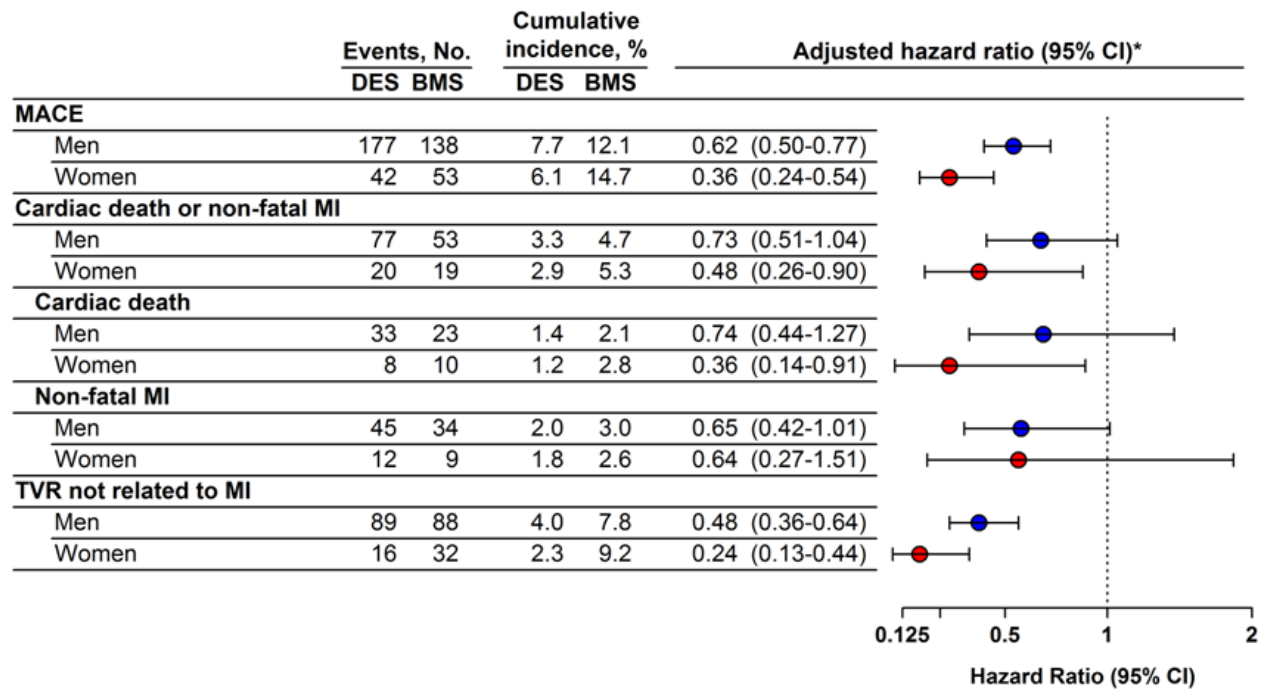

Figure 3 HRs endpoints at 2 years in women and men. ${ }^{*}$ The models for MACE were adjusted for age, clinical presentation, smoking, diabetes mellitus, hypertension and hypercholesterolaemia, while the remainders of endpoints were adjusted for age. BMS, bare-metal stent; DES, drug-eluting stent; MACE, major adverse cardiac events; MI, myocardial infarction; TVR, targetvessel revascularisation.

The composition of atherosclerotic plaques has been shown to differ between the sexes, and among patients with acute coronary syndrome less rupture and less necrotic core and calcium was seen by angiographic and intravascular ultrasound measures in women compared with men. ${ }^{15}$ Moreover, release and distribution of drugs from DES in vitro depends on the compositions of the atherosclerotic plaque. ${ }^{16}$ The plaque-dependent difference in drug distribution and diffusion to the vessel wall in combination with any sex-related plaques compositions may explain some of the differences in safety and efficacy between men and women treated with DES.

\section{Strengths and limitations}

By pooling data from two clinical trials with very similar design, follow-up time, endpoints and inclusion and exclusion criteria, we analysed a large, very homogenous study population from several European countries. Still, our study has some limitations. First, different types of DES were used in the two studies. Thus, only an overall effect of DES was examined and it was not possible to detect if one type of DES was more effective. Second, the prescribed antithrombotic medications were of different types in BP and BPII, respectively. Third, not enough safety events were found to allow meaningful interaction testing between sex and type of stent. Fourth, no quantitative data on coronary angiography findings were available to us. Finally, we were examining patients with large coronary vessels, and it is therefore not possible to extend the results to patients with smaller vessels.

\section{CONCLUSION}

In this pooled study of patients requiring stenting of large coronary arteries, we found evidence that DES was associated with improved safety in terms of cardiac death and non-fatal MI at 2 years compared with BMS in women. Efficacy of DES versus BMS was superior in both sexes, although women benefited more. Thus, DES is a safer and more effective stent in large coronary arteries in women than BMS and may be regarded as stent of choice for women needing stenting of large coronary arteries. Future studies should seek to elucidate the biological mechanisms underlying the suggested differential vascular responses to stent implantation in women and men.

\section{Author affiliations}

${ }^{1}$ Department of Cardiology, Bispebjerg Frederiksberg University Hospital, Copenhagen, Denmark

${ }^{2}$ Department of Cardiology, Rigshospitalet, Copenhagen, Denmark

${ }^{3}$ Department of Cardiology, Herlev Gentofte University Hospital, Hellerup, Denmark

${ }^{4}$ Department of Cardiology, University Hospital Basel, Basel, Switzerland

Acknowledgements The authors would like to thank all the investigators and participants of the BASKET PROVE and the BASKET PROVE II trials.

Contributors All authors have contributed significantly to the project to be included as authors, and participated in the making of the study design. LHB performed data analysis and wrote the manuscript. All authors interpreted the results, revised the manuscript, and approved the final version.

Funding This project was financed by Department of Cardiology, University Hospital Bispebjerg, Denmark. The BASKET-PROVE trial was funded by the Basel Cardiovascular Research Foundation, Basel, Switzerland and by the Swiss National Foundation for Research, Berne, Switzerland. The BASKET-PROVE II trial was funded by the Basel Cardiovascular Research Foundation, Basel, Switzerland.

\section{Competing interests None declared.}

Patient consent for publication Not required.

Provenance and peer review Not commissioned; externally peer reviewed.

Data sharing statement Data may be obtained from a third party and are not publicly available.

Author note Prasugrel was provided free of charge to all patients in BASKETPROVE II by Daiichy Sankyo and Eli Lilly.

Open access This is an open access article distributed in accordance with the Creative Commons Attribution Non Commercial (CC BY-NC 4.0) license, which permits others to distribute, remix, adapt, build upon this work non-commercially, 
and license their derivative works on different terms, provided the original work is properly cited, appropriate credit is given, any changes made indicated, and the use is non-commercial. See: http://creativecommons.org/licenses/by-nc/4.0/.

\section{REFERENCES}

1. Kirtane AJ, Gupta A, lyengar S, et al. Safety and efficacy of drug-eluting and bare metal stents: comprehensive meta-analysis of randomized trials and observational studies. Circulation 2009;119:3198-206.

2. Palmerini T, Benedetto U, Biondi-Zoccai G, et al. Long-Term Safety of Drug-Eluting and Bare-Metal Stents: Evidence From a Comprehensive Network Meta-Analysis. J Am Coll Cardiol 2015;65:2496-507.

3. Kaiser C, Galatius S, Jeger R, et al. Long-term efficacy and safety of biodegradable-polymer biolimus-eluting stents: main results of The Basel stent Kosten-Effektivitäts Trial-PROspective validation examination II (BASKET-PROVE II), a randomized, controlled noninferiority 2-year outcome trial. Circulation 2015;131:74-81.

4. Bønaa KH, Mannsverk J, Wiseth R, et al. Drug-eluting or bare-metal stents for coronary artery disease. N Engl J Med 2016.

5. Regueiro A, Fernández-Rodríguez D, Brugaletta S, et al. Sex-related impact on clinical outcome of everolimus-eluting versus baremetal stents in ST-segment myocardial infarction. Insights from the examination trial. Rev Esp Cardiol 2015;68:382-9.

6. Kornowski R, Vaknin-Assa $\mathrm{H}$, Assali A, et al. A comparative analysis of major clinical outcomes with drug-eluting stents versus bare metal stents in male versus female patients. Eurolntervention 2012;7:1051-9.
7. Hansen KW, Kaiser C, Hvelplund A, et al. Improved two-year outcomes after drug-eluting versus bare-metal stent implantation in women and men with large coronary arteries: importance of vesse size. Int J Cardiol 2013;169:29-34.

8. Stefanini GG, Baber U, Windecker S, et al. Safety and efficacy of drug-eluting stents in women: a patient-level pooled analysis of randomised trials. The Lancet 2013:382:1879-88.

9. Pfisterer M, Bertel O, Bonetti PO, et al. Drug-eluting or bare-metal stents for large coronary vessel stenting? The BASKET-PROVE (prospective validation examination) trial: study protocol and design. Am Heart J 2008;155:609-14.

10. Kaiser C, Galatius S, Erne P, et al. Drug-eluting versus bare-meta stents in large coronary arteries. N Engl J Med 2010;363:2310-9.

11. Jeger R, Pfisterer M, Alber $\mathrm{H}$, et al. Newest-generation drugeluting and bare-metal stents combined with prasugrel-based antiplatelet therapy in large coronary arteries: The Basel stent Kosten Effektivitäts trial prospective validation examination Part II (BASKETPROVE II) trial design. Am Heart J 2012;163:136-41.

12. Papakonstantinou NA, Stamou MI, Baikoussis NG, et al. Sex differentiation with regard to coronary artery disease. $J$ Cardiol 2013;62:4-11.

13. Kumar A, Kaur H, Devi P. Coronary artery disease in women: how does it differ from men? JIACM 2011:13:43-7.

14. Shaw LJ, Bugiardini R, Merz CNB. Women and ischemic heart disease: evolving knowledge. J Am Coll Cardiol 2009;54:1561-75.

15. Lansky AJ, Ng VG, Maehara A, et al. Gender and the extent of coronary atherosclerosis, plaque composition, and clinical outcomes in acute coronary syndromes. JACC Cardiovasc Imaging 2012;5(3 Suppl):S62-S72.

16. Guo J, Saylor DM, Glaser EP, et al. Impact of artificial plaque composition on drug transport. J Pharm Sci 2013;102:1905-14. 